\title{
Todos los números el número ${ }^{\uparrow}$
}

\section{All Numbers the Number}

\author{
Adolfo Yalta Dorregaray \\ Universidad de Lima
}

\section{RESUMEN}

El número es un concepto abstracto que apareció simultáneamente en el mundo, pero no de modo uniforme. Cada grupo humano tuvo una grafía particular para representarlos. Estos dialectos numéricos fueron desarrollándose hasta transformarse en el lenguaje universal conformado por el sistema numérico indoarábigo, conformado por diez símbolos: $0 ; 1 ; 2$; $3 ; 4 ; 5 ; 6 ; 7 ; 8$ y 9 o dígitos, en recuerdo de la base antropomórfica en la que se supone está basado (los diez dedos de las manos). El proceso de contar posiblemente haya sido una aplicación anterior a otras, según avanzaba el desarrollo tecnológico: medir, ordenar y codificar. Por eso, en el transcurso del tiempo, han ido apareciendo los números que se utilizan en la actualidad: enteros, racionales, irracionales, reales y complejos. Además, aparecen otros números reales cuyo interés es puramente matemático, como los números trascendentes, números algebraicos y números normales. Desde la antigüedad, los números naturales también han tenido una función lúdica de modo que, conforme a ciertos procesos de adición o multiplicación, se han definido los números primos, números perfectos, números amigos y una larga lista de números con nombres de fantasía. Sin embargo, no debe perderse de vista que la base para encriptar mensajes (función de codificación de los números) está basada en los números primos.

\section{Palabras clave:}

Número / Tipos de números (naturales, enteros, racionales, irracionales, reales y complejos) / Cardinal de un conjunto / Conjunto numerable / Conjunto no numerable / Números notables (primos, perfectos, amigos)

\section{ABSTRACT}

The number is an abstract concept that appeared in various geographical points of the planet almost simultaneously but not in a uniform way. Each human group had a particular script to represent them. These numerical dialects were developed until becoming the universal language conformed by the numerical system indoarabic, which is a positional system conformed by ten symbols: $0 ; 1 ; 2 ; 3$; $4 ; 5 ; 6 ; 7 ; 8$ and 9 . These symbols are called digits in memory of the anthropomorphic basis on which it is assumed to be based (the ten fingers of the hands). The process of counting may have been the primordial application that human beings made of the numbers. Then, as the technology that transformed the surrounding world developed, they used their other basic properties: measuring, ordering, and coding.

The numbers that are used to count were called natural numbers but to meet the demands of technological development are insufficient. For this reason, in the course of time, the numbers currently used - have appeared, that is to say whole, rational, irrational, real and complex. In addition, other real numbers appear, but their use is purely mathematical like the transcendent numbers, algebraic numbers and normal numbers. Since ancient times, natural numbers have also had a ludic function so that, according to certain processes of addition or multiplication, prime numbers, perfect numbers, friend numbers and a long list of numbers with fancy names have been defined. However, it should not be overlooked that the basis for encrypting messages (code encoding function) is based on prime numbers.

\section{Keywords:}

Number / Types of Numbers (Natural, Whole, Rational, Irrational, Real and Complex) / Cardinal of a Set / Numerable Set / Non-Countable Set / Notable Numbers (Primes, Perfects, Friends).

1 El título es un homenaje a Julio Cortázar por el libro Todos los fuegos el fuego (1966). 


\section{Introducción al mundo del número}

E I reconocido matemático contemporáneo lan Stewart (2009), autor de varios libros de divulgación de la matemática, afirma que sin los números la civilización, tal como la conocemos, no podría existir. Mario Livio (2009) escribe que el filósofo francés René Descartes (1596-1650) quería que las explicaciones científicas estén dadas en el lenguaje matemático y por esto, Descartes afirmaba lo siguiente: "No reconozco sustancia alguna en las entidades corpóreas salvo lo que los geómetras llaman cantidad y convierten en el objeto de sus demostraciones...Y, siendo que todos los fenómenos naturales pueden explicarse de este modo, sostengo que ningún otro principio es admisible o siquiera deseable en física"2. Por otro lado, el filósofo escocés David Hume (1711-1776), citado por Jesús Mosterín (2008), escribe acerca del entendimiento humano: "Si cogemos en nuestras manos un libro cualquiera..., preguntémonos, ¿contiene algún razonamiento abstracto sobre la cantidad o el número? No. ¿Contiene algún razonamiento experimental sobre asuntos de hecho y de existencia? No ${ }^{3}$. Entonces arrojémoslo a las Ilamas pues no puede contener sino sofismas ${ }^{4}$ y engaños". Estas afirmaciones son muy radicales, pero sirven para resaltar, de algún modo, la importancia que ha tenido el número en el desarrollo de nuestra civilización. Y si no, realice el siguiente ejercicio mental: imagine que un día despierta y se encuentra en un mundo donde no existen ni los números ni los pensamientos numéricos. El desorden y el caos se instalarían no solo en su vida, sino en toda la sociedad a la cual pertenece, porque se habrían perdido las cuatro funciones básicas utilizadas del número, como son contar, ordenar, medir y codificar las cosas. Todo sería muy complicado y el mundo sería muy diferente al que conocemos: una pesadilla. Cabe indicar que las dos primeras funciones del número, contar y ordenar, aparecieron casi con los números mientras que la tercera, medir, aparece por la necesidad de comparar las magnitudes asociadas a lo que rodea al hombre con un patrón-unidad. La última, codificar, es la función numérica que recién ha aparecido pero que está adquiriendo una gran importancia debido a la tecnología que actualmente impera en nuestra civilización.

Parece que, en base a esta posible pesadilla, el escritor de temas científicos Isaac Asimov, en su libro De los números y su historia (1988), decía "No creo que haya hoy sobre la Tierra una tribu de seres humanos, por más primitiva que sea, que no tenga alguna noción del número". Pero esta afirmación es negada por la realidad, puesto que, tanto en la antigüedad como en nuestra época, han existido y existen grupos humanos para los cuales la pesadilla mencionada no tiene ninguna razón de ser ya que no tienen desarrollado el concepto del número. Daniel Tammet (2015) señala que la tribu amazónica brasileña de los piraha es una sociedad que vive totalmente al margen de los números. El lenguaje con el que se comunican tiene solo ocho consonantes y tres vocales y carecen de numerales. La información que se conoce sobre esta tribu se debe al lingüista californiano Daniel Everett, que ha dedicado treinta años de su vida a estudiarlos, conviviendo con ellos. En el mismo libro, Tammet agrega que entre las culturas que manejan pocos números están los caquintes del Perú, quienes cuentan uno (aparo) y dos (mavite), al tres lo llaman "es otro más", al cuatro "el que le sigue"; los veddas una tribu indígena de Sri Lanka, usa palabras solamente para el uno (ekkamal) y el dos (dekkamal) porque para cantidades mayores continúan "y uno más, y uno más, y uno más...". Estos grupos humanos son minoritarios frente al resto de culturas del mundo porque muchos pueblos como los sumerios, indios, chinos, europeos y mayas, entre otros, sí desarrollaron el concepto de número de una manera clara y precisa, incluyendo su simbolización la cual, evidentemente, era muy propia de cada cultura. Puede afirmarse que los números aparecieron universalmente en la antigüedad, pero no de forma uniforme.

Desde su aparición, los números se han aplicado a la vida cotidiana. Al parecer su primera aplicación fue contar cosas. ¿Cuántos son los miembros de la tribu? (la Biblia contiene un libro denominado Números, en el que se presenta la cantidad de integrantes de cada tribu del pueblo israelita, además de otros temas referentes a la doctrina cristiana), ¿cuántos animales de caza se han avistado? ¿ ¿cuántos días faltan para que vuelva a aparecer la luna llena? Para esos grupos humanos conocer las respuestas a estas preguntas era, a veces, cuestión de vida o muerte. Por ejemplo, la respuesta a la pregunta ¿cuántas fieras salvajes vienen?, era la diferencia entre tener comida o ser comida. En el mismo ejemplo, ¿quién da la orden de atacar? ¿Quién dispara primero? ¿Quién recibe el primer trozo de carne? La aparición de la jerarquización en la realización de las actividades significó el surgimiento de un ordenamiento: primero fulano, luego mengano...

Estos procesos de contar y ordenar se tornaron algo común y aceptado por los grupos humanos cuando dejaron la etapa de cazador-recolector para iniciar la de agricultor-ganadero ya que, al establecerse en un lugar, tenían necesidad de conocer cuántos animales conformaban su rebaño o cada cuántos días debían sembrar o cosechar. Además, apareció la necesidad de medir los terrenos que pasaron de ser comunales a ser propiedad individual. Esto es, los números se utilizaban no solo para contar y ordenar, sino también para medir.

3 David Hume, en Investigación sobre el conocimiento humano (1748).

4 Sofisma: Argumento falso, presentado para inducir al error. Sinónimos: Argucia, engaño, falsedad. 
Al principio no existían símbolos para representar los números. Por ejemplo, un pastor no tenía forma de conocer, en todo momento, cuántas ovejas conformaban su rebaño. Hasta que alguno creativo estableció una relación entre las ovejas del rebaño y un montón de piedrecillas ${ }^{5}$, de modo que a cada oveja le correspondía una piedrecilla y a cada piedrecilla le correspondía una oveja. Este primitivo proceso de contar ha quedado perennizado por la palabra "cálculo", que corresponde a una parte importante de la matemática, ya que proviene del latín calculus, que significa piedra. En la matemática actual, al proceso de relacionar cada oveja con una piedrecilla se le denomina correspondencia biunívoca entre dos conjuntos (0 relación uno-a-uno). Más adelante, se indica como el matemático alemán George Cantor (1848-1918) utilizó esta relación para presentarnos los conjuntos numerables, conjuntos no numerables y, luego, los números transfinitos.

Para contar, además de los montones de piedrecillas, se utilizaban los dedos, marcas en palos o huesos (Hueso de Ishango ${ }^{6}$ ), nudos en cuerdas (quipus ${ }^{7}$ ) y algunas otras maneras ingeniosas de ir contabilizando las cosas. La forma más simple era añadir una señal tras otra sin importar su forma. Pero esto era tan engorroso como cargar consigo el montón de piedrecillas. En casi todas las culturas, y en diferentes épocas, la creatividad del ser humano simplificó este proceso, al establecer que luego de cierta cantidad de señales, se utilizara otra señal diferente, de manera que representara a todos los objetos contabilizados hasta esa parte. La cantidad señalada es lo que actualmente se conoce como base del sistema de numeración utilizado. Las bases más usadas en el mundo antiguo tenían referencias antropomórficas: cinco (por los dedos de una mano), diez (los dedos de las dos manos), veinte (los dedos de las manos y de los pies). Por esta razón, en la matemática se utiliza la palabra "dígito", que proviene del latín digitus que significa 'dedo', como sinónimo de número que se expresa con un solo símbolo. Algunas excepciones fueron los babilonios en la región mesopotámica, que usaban el 10 y el 60 como base, y los mayas en América, quienes usaron el 5 y 20 como base. En la actualidad, la base 60 se sigue utilizando tanto en la medida del tiempo (una hora se divide en 60 minutos y un minuto en 60 segundos), como en la medida de los ángulos mediante el sistema sexagesimal (un ángulo sexagesimal se divide en 60 ángulos de 1 minuto y cada uno de estos ángulos se dividen en 60 ángulos de 1 segundo).

En este artículo no se pretende relatar la historia de los números, sino presentar una clasificación muy sucinta de los mismos, así como destacar algunos de ellos. Con esto en mente, nos referiremos, a continuación, al sistema de numeración que utilizamos en la actualidad. A este sistema numérico no se llegó directamente sino a través de los meandros de los descubrimientos científicos, con avances y retrocesos, con inspiración y, evidentemente, mucha transpiración.

Este sistema de numeración es posicional de base 10 y cuenta con los dígitos $0 ; 1 ; 2 ; 3 ; 4 ; 5 ; 6 ; 7 ; 8$ y 9 . Fue creado en la India pero, por haber sido popularizado por los árabes, se les denomina números arábigos. Aunque fue Leonardo de Pisa, más conocido como Fibonacci, quien, al publicar el libro Liber abaci (Libro del ábaco o Libro del cálculo), en 1202, Ios hizo conocer en Europa. Fibonacci mostró la importancia del nuevo sistema de numeración, que facilitaba los cálculos que se utilizaban tanto en la contabilidad comercial (transacciones comerciales, cambio de una moneda a otra) como en la conversión de las diferentes medidas de longitud, área y volumen usadas en la época. Pero en sus páginas, no solo mostró la parte utilitaria del nuevo sistema de numeración, sino que presentó la descomposición de un número en sus factores primos y criterios de divisibilidad, entre otros resultados matemáticos. La popularidad del nuevo sistema numérico creció a gran velocidad entre las personas cultas y, especialmente, entre los comerciantes y banqueros.El prolífico y excéntrico matemático húngaro Paul Erdös (1913-1996) escribió lo siguiente acerca de la belleza de los números: "Es como preguntar por qué la Novena Sinfonía de Beethoven es bella. Si no ve que es así, nadie se lo puede explicar. Yo sé que los números son bellos, si no lo son, nada lo es". Y como la poesía es la manifestación de la belleza a través de la palabra, entonces no puede estar ajena a este influjo numérico. Por ello, los poetas españoles Rafael Alberti (1902-1999) y José Verón (1946), así como el poeta chileno Pablo Neruda (1904-1973), entre otros poetas, dedican unos versos muy sentidos, el primero en El ángel de los números, el segundo en la Balada de los números, y el tercero en dos poemas: Oda a los números y el titulado Por su brevedad y belleza poética, presentamos el poema de Alberti: Vírgenes con escuadras/y compases, velando/ las celestes pizarras. / Y el ángel de los números, /pensativo, volando del 1 al 2, del 2/al 3, del 3 al 4. /Tizas frías y esponjas/ rayaban y borraban/la luz de los espacios. /Ni sol, luna, ni estrellas, /ni el repentino verde/del rayo y el relámpago, / ni el aire.

5 Homero, en su libro La Odisea, cuenta que el gigante Polifemo, enceguecido por Ulises, hacía lo mismo que el pastor creativo: sentado cada mañana, a la entrada de su cueva, controlaba el ingreso y salida de las ovejas de su rebaño mediante un grupo de piedras. La historia de Polifemo es una de las referencias literarias más antiguas de la correspondencia biunívoca entre los objetos que forman los conjuntos de ovejas y de piedras.

6 El Hueso de Ishango es un hueso de babuino con diversas marcas, hallado por el explorador belga Jean de Heinzelin, en 1960, en una zona que hoy pertenece a la República Democrática del Congo. Ha sido estudiado por diferentes científicos, quienes han señalado que esas marcas indican alguna destreza matemática que va más allá del proceso de contar.

7 Indican los historiadores de la época incaica que los quipus están de alguna manera relacionados con el sistema de contabilidad de la administración del imperio incaico. Es decir, con los procesos de contar. 
Sólo nieblas. / Vírgenes sin escuadras, / sin compases, llorando. / $\mathrm{Y}$ en las muertas pizarras, /el ángel de los números, /sin vida, amortajado /sobre el 1 y el 2, / sobre el 3, sobre el 4 ...

Realmente, como podemos ver, los números son omnipresentes: están en casi todas las actividades que realizamos.

Antes de presentar una clasificación de los números, procuremos responder la pregunta crucial: ¿Qué es el número? Recurriendo al Diccionario de la Real Academia de la Lengua Española (DRAE) se encuentran catorce acepciones correspondientes a número, de las cuales solamente la primera (expresión de una cantidad con relación a su unidad) es la que más se acerca al sentido que se le da al número en la matemática. Pero la respuesta no es fácil, porque, como señala lan Stewart (2009), “...Los cálculos con números pueden ser duros; obtener el número correcto puede ser difícil. Incluso así, es mucho más fácil utilizar números que especificar qué son realmente". Más adelante, en el mismo texto, indica que "El significado de 'número' es un problema filosófico y conceptual sorprendentemente difícil. Todo es más frustrante por el hecho de que todos conocemos perfectamente cómo utilizar los números. Sabemos cómo se comportan, pero no sabemos lo que son". De este modo, lan Stewart adopta la misma posición que San Agustín respecto de la pregunta: ¿Qué es, pues, el tiempo? Parafraseando al filósofo católico, parece que nos invita a decir: "Si nadie me pregunta qué es el número, lo sé; pero si quiero explicárselo al que me lo pregunta, no lo sé". Por otro lado, el matemático alemán Richard Dedekind (1831-1916), en la monografía ¿Qué son y para qué sirven los números? (1887) afirma que los números son creaciones libres del espíritu humano, sirven como medio para concebir más fácil y claramente la diversidad de las cosas. A continuación, asevera que la ciencia de los números debe erigirse sobre el fundamento de relacionar cosas con cosas, hacer corresponder una cosa a otra o representar una cosa mediante otra. El fundamento mencionado por Dedekind es la base para que el matemático alemán Gottlob Frege (1848-1925) presente en su libro Los fundamentos de la aritmética una definición de número natural a partir del concepto de conjunto del siguiente modo: el número natural es el conjunto de todos los conjuntos para los que puede establecerse una correspondencia uno-a-uno con el conjunto estándar $\left\{\mathrm{a}_{1} ; \mathrm{a}_{2} ; \ldots ; \mathrm{a}_{\mathrm{n}}\right\}$. Así, el número 2 es el conjunto $\{\ldots\{m ; n\},\{$ perro; gato $\},\{X ; Y\},\{A$ y $B$ son los extremo del segmento $A B\},\{\pi ;-1\}, \ldots\}$

Para Dedekind y Frege los números naturales son los cardinales de los conjuntos finitos y, por eso, Joaquín Navarro (2011) presenta la siguiente definición moderna del número natural de modo recursivo:

$0=\{\because\} ; 1=\{0\} ; 2=\{0 ; 1\} ; 3=\{0 ; 1 ; 2\} ; 4=\{0 ; 1 ; 2 ; 3\} ; \ldots ; n=\{0 ; 1 ; 2 ; 3 ; \ldots n-1\}$

Debe destacarse que el conjunto se denomina conjunto vacío porque no tiene ningún elemento y fue el matemático francés André Weil (1906-1998), quien sugirió la letra danesa $\emptyset$ para representarlo. Parece que el símbolo escogido por Weil sigue la regla no escrita de colocar una diagonal sobre lo que un símbolo afirma, para expresar su negación: $\in$ (pertenece), $\notin$ (no pertenece); = (igual), $\neq$ (no es igual); $\subset$ (incluido), $\not \subset$ (no incluido) y, en este caso, 0 (universo), $\varnothing$ (vacío).

\section{Clasificación de los números}

La clasificación de los números, que a continuación se presenta, está basada en la forma actual de presentación de los números en el mundo académico: números naturales, números enteros, números racionales, números irracionales, números reales y números complejos.

- Los números naturales son los números por antonomasia: en la actualidad, cada vez que una persona piensa en un número piensa en un número natural... de una manera natural. De ahí su nombre: naturales. Estos números son usados para contar objetos; por esto, el pastor de las piedrecillas los utilizaba para contar sus ovejas, sin saber que eran números naturales. El conjunto de los números naturales se denota con $\mathbb{N}$ (letra inicial de natural) y se presenta del siguiente modo: $\mathbb{N}=\{0 ; 1 ; 2 ; 3 ; 4 ; .$.$\} , donde los tres puntos suspensivos significa que los números naturales continúan$ hacia la derecha, agregándole una unidad al número de la izquierda.

8 “Qué es, pues, el tiempo? Si nadie me lo pregunta, lo sé; pero si quiero explicárselo al que me lo pregunta, no lo sé. Lo que sí digo sin vacilación es que sé que si nada pasase no habría tiempo pasado; y si nada sucediese, no habría tiempo futuro; y si nada existiese, no habría tiempo presente. Pero aquellos dos tiempos, pretérito y futuro, ¿cómo pueden ser, si el pretérito ya no es y el futuro todavía no es? $Y$ en cuanto al presente, si fuese siempre presente y no pasase a ser pretérito, ya no sería tiempo, sino eternidad. Si el presente, para ser tiempo, es necesario que pase a ser pretérito, ¿cómo deciros que existe éste, cuya causa o razón de ser está en dejar de ser, de tal modo que no podemos decir con verdad que existe el tiempo sino en cuanto tiende a no ser?" San Agustín de Hipona en Confesiones (400 d.C.). 
- La adición y multiplicación de dos números naturales es siempre un número natural. Esta propiedad se denomina clausura. Pero la sustracción, división y radicación de números naturales no verifica esta propiedad, ya que los resultados correspondientes a estas operaciones no siempre son números naturales. Por ejemplo, no son números naturales las respuestas que corresponden a las operaciones $2-4 ; 5 / 3$ y $\sqrt[3]{16}$.

Cabe resaltar que los números naturales se utilizan para determinar la cantidad y el orden de las cosas del mundo. En el primer caso, se dice que se trata de números cardinales y, en el segundo, de números ordinales. Por ejemplo, el símbolo 2 se lee "dos" en los números cardinales y con él nos estamos refiriendo a dos cosas cualesquiera (dos amigos, dos proposiciones). Sin embargo, este mismo 2 se lee "segundo" en los números ordinales y estaremos indicando que lo señalado con 2 ocupa la segunda posición.

Si recordamos el proceso de comparar piedrecillas con las ovejas parece ser que los números cardinales aparecieron antes que los números ordinales porque había la necesidad de contar. Pero algunos estudiosos del tema afirman que, en los grupos humanos antiguos, por cuestiones religiosas, antes que la cantidad de objetos estuvo el orden de prelación acerca de quién es primero, quién es segundo y así sucesivamente. En otras palabras, no se puede precisar cuál de estos tipos de números aparecieron en los albores de la civilización.

- L Los números enteros son una ampliación de los números naturales y se los utiliza para expresar la posición relativa del número con respecto al cero. Al conjunto de los números enteros se le denota con $\mathbb{Z}$ (letra inicial de la palabra alemana Zahlen, que significa números) y se presenta de este modo $\mathbb{Z}=\{. . . ;-2 ;-1 ; 0 ; 1 ; 2 ; . .$.$\} , donde los puntos suspensivos$ significan que los números enteros continúan hacia la izquierda y hacia la derecha.

La adición, multiplicación y sustracción de dos números enteros verifican la propiedad de clausura. Pero la división y radicación de números enteros no verifica dicha propiedad. Por ejemplo, no son números enteros ni 2/3 ni $\sqrt{2}$.

El número de elementos de un conjunto se denomina cardinal del conjunto y se denota por $\operatorname{card}(A)$. Los conjuntos finitos tienen por cardinal un número natural. En cambio, para el cardinal de un conjunto infinito el matemático alemán George Cantor (1845-1918) creó los números transfinitos (más allá de lo finito), simbolizándolos por la primera letra del alfabeto hebreo $\mathrm{N}$ (aleph) acompañada de un subíndice: $\aleph_{0}, \aleph_{1}, \aleph_{2}, \aleph_{3}, \ldots$. llamados aleph-0, aleph-1, aleph-2, aleph-3... El menor de los números transfinitos es el cardinal de $\mathbb{N}$. Esto es, $\operatorname{card}(\mathbb{N})=\aleph_{0}$. Su creador presentó una aritmética de los números transfinitos, destacándose, entre otras operaciones, las siguientes: $\aleph_{0}+\aleph_{0}=\aleph_{0}, \aleph_{0}+\aleph_{1}=\aleph_{1}$, $\aleph_{1}+\aleph_{2}=\aleph_{2}, \aleph_{0}, \aleph_{0}=\aleph_{0}$.

Obsérvese que todo número natural es un número entero, pero existen números enteros que no son números naturales. En las matemáticas esta afirmación se simboliza como $\mathbb{N} \subset \mathbb{Z}$ y se lee: $\mathbb{N}$ está incluida en $\mathbb{Z}$ y parece obvio que el cardinal de $\mathbb{N}$ debe ser menor que el de $\mathbb{Z}$ Pero esto, como veremos más adelante, no es cierto.

Cantor denominó conjunto numerable, o contable, a un conjunto $A$ de infinitos elementos tal que entre los elementos del conjunto $A$ y los números naturales puede efectuarse una relación uno-a-uno. Además, indicó que, en este caso, se cumple card $(A)=\aleph_{0}$. Mostró que los siguientes subconjuntos de $\mathbb{N}$ impares, pares, primos, múltiplos de 4, potencias de 2, entre otros subconjuntos, son numerables, porque a cada uno de esos subconjuntos se le puede aplicar una correspondencia uno-a-uno con $\mathbb{N}$. La existencia de esta relación contradice la afirmación euclidiano-aristotélica de que el todo es mayor que cualquiera de sus partes, porque, en los casos mencionados, el todo, $\mathbb{N}$, tiene tantos elementos como cualquiera de los subconjuntos indicados. Cabe señalar que un resultado similar ya había sido obtenido por el astrónomo, físico, matemático y filósofo italiano Galileo Galilei (1564-1642), quien, en Diálogos sobre los dos máximos sistemas del mundo (1632), señalaba que entre los números naturales $\eta$ y sus cuadrados $\eta^{2}$ podía establecerse una relación uno-a-uno, de modo que no podía afirmarse que el todo era mayor que una de sus partes. Pero Galileo aducía que los atributos de "igual, "mayor" y "menor" no tienen lugar en los infinitos, sino solo en las cantidades finitas.

Sin embargo, Cantor demostró que $\mathbb{Z}$ es numerable porque a cada número entero se le puede asociar un único número entero natural. Con los números naturales y números enteros hizo lo mismo que el pastorcillo con sus ovejas y las piedrecillas, pero de una manera muy ingeniosa: el 0 entero lo asoció con el 0 natural $(0 \leftrightarrow 0)$, cada número entero positivo lo asoció con un número natural impar $(1 \leftrightarrow 1 ; 2 \leftrightarrow 3 ; 3 \leftrightarrow 5 ; . .$.$) y cada número entero negativo lo asoció con un$ número natural par $(-1 \leftrightarrow 2 ;-2 \leftrightarrow 4 ;-3 \leftrightarrow 6 ;-4 \leftrightarrow 8$ : ...). De este modo, como a cada número entero le corresponde un único número natural, entonces $\mathbb{Z}$ es numerable. $Y$ si es numerable se verifica que $\operatorname{card}(\mathbb{Z})=\aleph_{0}$. Por lo tanto, $\operatorname{card}(\mathbb{Z})=\operatorname{card}(\mathbb{N})=\aleph_{0}$ 
Hay que resaltar que los conjuntos $\mathbb{N}$ y $\mathbb{Z}$ son conjuntos discretos porque entre dos elementos contiguos no existe un elemento intermedio. Por ejemplo, entre 5 y 6 no existe un número natural intermedio, así como entre -1 y 0 no se encuentra un número entero.

- Los números racionales son aquellos que se escriben como el cociente de dos números enteros en los que el divisor no es cero. El nombre de raciona/ viene de "razón" que en matemáticas es sinónimo de cociente o división o fracción. Cabe resaltar que la notación decimal de los números racionales, tal como en la actualidad se usa, se basó en el libro De Thiende (El decimal), escrita por el matemático belga Simón Stevin (1548-1620), alrededor de 1585. En el libro se presentó tanto la notación decimal como la forma de realizar cálculos con la misma.

Al conjunto de los números racionales se le denota con $\mathbb{Q}$ (letra inicial de la palabra inglesa quotient). Se presenta del siguiente modo: $\mathbb{Q}=\{p / q / p \in \mathbb{Z}, q \in \mathbb{Z}, q \neq 0\}$. Se cumple que $\mathbb{N} \subset \mathbb{Z} \subset \mathbb{Q}$. Esto es, los números naturales y números enteros son números racionales porque basta con presentarlos como fracciones con denominador igual a 1. Sin embargo, existen números racionales que no son ni naturales ni enteros, como 0,24 0-13/11. Algunos números racionales son $1 / 2 ; 666 ; 3,1416 ; 5 \frac{4}{7} ;-\frac{7}{3} ; 1,2=1,222 \ldots=11 / 9$.

La adición, multiplicación, sustracción y división de dos números racionales verifican la propiedad de clausura. Pero la radicación de números racionales no la verifica, porque algunos radicales no corresponden a fracciones. Por ejemplo, $\sqrt{2}, \sqrt[3]{9}$ y $\sqrt{-2}$ no son números racionales.

El conjunto $\mathbb{Q}$, a diferencia de los conjuntos $\mathbb{N}$ y $\mathbb{Z}$, no es un conjunto discreto, sino que es un conjunto denso, ya que entre dos números racionales siempre podemos hallar otro número racional: bastará con determinar la semisuma de los mismos. Así, entre 1/3 y 1/2 se encuentra 5/12 porque es la semisuma de los mismos. Y entre 1/3 y 1/2, por el mismo procedimiento, se encuentra $3 / 8$. Es evidente que este proceso no tiene fin. Consecuentemente, un segmento de recta numérica, por pequeño que sea, contiene infinitos números racionales y algunos números enteros o ninguno. A pesar de esto, Cantor demostró que el conjunto $\mathbb{Q}$ es numerable. La forma como construyó la correspondencia uno-a-uno entre los números naturales y los números racionales es una muestra del genio matemático de Cantor, quien procedió del siguiente modo: colocó en una línea las fracciones que están formadas por dos números naturales, excluyendo al cero como denominador, tomando en cuenta la suma de su numerador y de su denominador. Comenzó con la única fracción cuya suma de sus componentes es igual a 1, la cual es 0/1. Siguió con otra única fracción cuya suma de sus componentes es 2 , que es 1/1. Luego, las fracciones en las que esta suma es 3 , que son 1/2 y 2/1 Continuó con las fracciones, cuya suma de componentes es 4 , que son 1/3 y 3/1 (no tomó en cuenta 2/2 porque es igual a que ya fue considerada) y prosiguió con las fracciones cuya suma es luego, las fracciones cuya suma es igual a 6, y así sucesivamente, pero sin considerar a aquellas fracciones que son iguales a alguna fracción que ya apareció en la lista. Obtuvo la siguiente sucesión de números racionales:

$0 / 1,1 / 1,1 / 2,2 / 1,1 / 3,3 / 1,1 / 4,2 / 3,3 / 2,1 / 5,5 / 1,1 / 6,2 / 5,3 / 4,4 / 3,5 / 2,6 / 1 \ldots$

Cualquier número racional aparecerá en la sucesión. Para esto bastará con prolongarla convenientemente. Para que aparezcan todos los racionales incluyó los racionales negativos, colocándolos alternamente con los racionales de la lista obtenida anteriormente:

$0 / 1,1 / 1,-1 / 1,1 / 2,-1 / 2,2 / 1,-2 / 1,1 / 3,-1 / 3,3 / 1,-3 / 1,1 / 4,-1 / 4,3 / 4,-3 / 4$

y luego, esta última lista la emparejó con los números naturales, como se muestra a continuación:

$\begin{array}{cccccccccccc}0 / 1 & 1 / 1 & -1 / 1 & 1 / 2 & -1 / 2 & 2 / 1 & -2 / 1 & 1 / 3 & -1 / 3 & 3 / 1 & -3 / 1 & \ldots \\ \uparrow & \uparrow & \uparrow & \uparrow & \uparrow & \uparrow & \uparrow & \uparrow & \uparrow & \uparrow & \uparrow & \\ 0 & 1 & 2 & 3 & 4 & 5 & 6 & 7 & 8 & 9 & 10 & \ldots\end{array}$

De este modo, Cantor mostró que card $(\mathbb{Q})=,\aleph_{0}$. En consecuencia, los números naturales, enteros y racionales son conjuntos numerables y tienen la misma cantidad de elementos: $\operatorname{card}(\mathbb{N})=\operatorname{card}(\mathbb{Z})=\operatorname{card}(\mathbb{Q})=,\aleph_{0}$.

- $\quad$ Los números irracionales son números que no pueden escribirse como una razón de números enteros (de ahí su nombre número irracional: número que no es racional).

A este conjunto se le denota con $\mathbb{I}$ (letra inicial de la palabra irracional) y, conforme a su nombre, se le presenta como $\mathbb{I}=\{\mathrm{x} / \mathrm{x} \neq(\mathrm{p} / \mathrm{q}), \mathrm{p} \in \mathbb{Z}, \mathrm{q} \in \mathbb{Z}, \mathrm{q} \neq 0\} \quad 0$, a veces, como $\mathbb{I}=\{\mathrm{x} / \mathrm{x}$ tiene una expresión decimal infinita y no periódica $\}$ 
porque no existe un número irracional que a la vez sea racional, y los números racionales se caracterizan por tener una expresión finita o infinita periódica. Por esto, en la teoría de conjuntos se afirma que $\mathbb{Q} \cap \mathbb{I}=\varnothing$ y se dice que son conjuntos disjuntos.

Algunos números irracionales son: e; $\pi ; \varphi=\frac{1+\sqrt{5}}{2}$ (número de oro) $; \sqrt{3} \mathrm{y} \sqrt[3]{-16}$.

El conjunto II no es numerable, como se verá más adelante.

Las operaciones de adición, multiplicación, sustracción, división y radicación de números irracionales no verifican la propiedad de clausura, porque, por ejemplo, las respuestas a las operaciones $\sqrt{2}+(-\sqrt{2}),(1 \sqrt{6})(1 / \sqrt{6}), \pi-\pi$ y $e / \pi$ no son números irracionales, sino números racionales.

La radicación de números irracionales tampoco verifica la propiedad de clausura, ya que, por ejemplo, $\sqrt{-\sqrt{2}}$ no pertenece a II porque no existe ningún número irracional cuyo cuadrado sea igual a $-\sqrt{2}$.

La presencia de los números irracionales en la matemática es tan antigua como algunos historiadores de esta ciencia lo afirman (parece que los babilonios ya los conocían). No obstante, es un lugar común decir que aparecieron con la escuela pitagórica. Incluso, se le atribuye a uno de sus integrantes, Hippasus de Metaponto, el descubrimiento del primer número irracional, $\sqrt{2}$, el cual, irónicamente, apareció al aplicar el teorema de Pitágoras para calcular la longitud de la diagonal de un cuadrado cuyo lado medía una unidad de medida. Este descubrimiento contradecía la esencia del pensamiento de Pitágoras y de sus seguidores, quienes sostenían que todo está dispuesto según el número, pero esta afirmación la realizaban refiriéndose a los números enteros y números racionales. Hippasus fue invitado por algunos colegas pitagóricos a un paseo mar adentro, para, supuestamente, celebrar su descubrimiento. Pero como el objetivo era silenciarlo para que no lo hiciera público, del paseo Hippasus no volvió. Una aplicación más de si no te gusta el mensaje, elimina al mensajero. Y, como siempre, la eliminación es inútil, porque si el mensaje tiene valor de todos modos prevalecerá: Ios irracionales irrumpieron en la matemática para quedarse y se demuestra, de una manera sencilla, que hay más números irracionales que números racionales.

Los números racionales, los que presentan periodos decimales, y los números irracionales, tienen una diferencia musical, porque si se asigna una nota a cada dígito del número racional se oiría un sonido que se repite, como un estribillo, mientras que para el número irracional, por la aparición desordenada de sus dígitos, las notas aparecerían sin formar una melodía.

- Los números reales no son números diferentes a los que hasta ahora han sido presentados, sino que es un nombre común a todos ellos. Esto es, los números naturales, enteros, racionales e irracionales son números reales. Una caracterización común a los números reales, entre otras, estriba en representarlos en forma decimal, conforme al método de escritura numérica creada por Simón Stevin, tal como se ha indicado anteriormente. Los números naturales y números enteros tienen la representación decimal más sencilla porque después de la coma decimal no tienen dígitos diferentes de cero. Los números racionales tienen una representación decimal conformada por infinitos dígitos, pero que, después del punto decimal, a partir de un dígito determinado, se repite un grupo de cifras una y otra vez (la cifra que se repite se denomina periodo decimal). Los números irracionales también tienen una representación decimal conformada por infinitos dígitos, pero, a diferencia de los números racionales, no poseen periodo. El conjunto de los números reales se denota por $\mathbb{R}$ (letra inicial de la palabra real) y, conforme a lo indicado en el párrafo anterior, se afirma que $\mathbb{R}=\mathbb{Q} \cup \mathbb{I}$ debido a que un número real cualquiera o tiene una presentación decimal periódica (número natural, entero o racional) o tiene una presentación decimal no periódica (número irracional).

George Cantor demostró que $\mathbb{N}, \mathbb{Z}$ y $\mathbb{Q}$ son conjuntos numerables, tal como se ha precisado anteriormente y, mostrando su creatividad matemática, probó que el conjunto $\mathbb{R}$ no es numerable. Su razonamiento es tan original que en la matemática se le denomina demostración diagonal o razonamiento diagonal, el cual, en esencia, es una demostración por reducción al absurdo, que es un argumento ampliamente utilizado en la demostración de teoremas en matemáticas9. Por esto, como Cantor afirmaba que el conjunto no es numerable, entonces, usando el método mencionado, supuso que es numerable. Luego, a partir de esta suposición, presentó una correspondencia uno-a-uno entre todos los números naturales y todos los números reales. A continuación, construyó un número real que no se encuentra en la correspondencia presentada ya que no puede emparejarse con ningún número natural. En consecuencia, es falso que

9 Este argumento consiste en demostrar que una proposición matemática es verdadera, probando que si fuera falsa entonces conduciría a una contradicción con alguna proposición verdadera previamente establecida. 
sea numerable: Esto es, $\mathbb{R}$ no es numerable. Conforme a Piñeiro (2013), se presenta un ejemplo con el cual se muestra el proceso seguido por Cantor para mostrar que no es numerable. La relación se presenta hasta el número natural 6 pero la lista, evidentemente, continúa indefinidamente:

$\begin{array}{cccc}\mathbb{N} & & \begin{array}{c}\mathbb{R} \text { (número real con } \\ \text { escritura decimal) }\end{array} & \begin{array}{c}\mathbb{R} \text { (número real sin } \\ \text { escritura decimal) }\end{array} \\ 0 & \leftrightarrow & 4,66666666 \ldots & (14 / 3) \\ 1 & \leftrightarrow & -2,19919919 \ldots & (-2197) / 999 \\ 2 & \leftrightarrow & 3,14159265 \ldots & \pi \\ 3 & \leftrightarrow & 12,00000000 \ldots & 12 \\ 4 & \leftrightarrow & 1,41421356 \ldots & \sqrt{2} \\ 5 & \leftrightarrow & -1,00000000 \ldots & -1 \\ 6 & \leftrightarrow & 0,69696969 \ldots & 23 / 35 \\ . & & \vdots & \end{array}$

La forma como se ha establecido la relación entre los números de los dos conjuntos es irrelevante, porque el razonamiento diagonal que utilizó Cantor se aplica sin importar cuál sea la regla de asignación. Solamente interesan los dígitos que están después de la coma decimal: se han puesto en negrita los dígitos que se encuentran en la diagonal que comienza en el extremo superior izquierdo y continúa descendiendo hacia la derecha (en el ejemplo la diagonal comienza con 6 y luego siguen $9,1,0,1,0,6, \ldots)$. Ahora, el número real $x$ que se está buscando, el cual queda fuera de la relación, puede tener cualquier parte entera, por ejemplo 4, y sus dígitos, después de la coma decimal, estarán relacionados con los que aparecen en la diagonal del siguiente modo: el primer dígito decimal de $x$ es igual a la primera cifra de la diagonal más el segundo dígito decimal de es el segundo dígito de la diagonal al cual se le suma 1 y se continúa sucesivamente (si en algún paso la suma es 10 entonces se escribe 0 en el lugar correspondiente de $x$ ). En este ejemplo el número real $x$ empieza con 4,7021217 ... y no le corresponde ningún número natural, debido a que no es el asignado al número 0, porque ambos difieren en el primer dígito decimal; ni al asignado al 1, ya que difieren en el segundo dígito. Tampoco puede ser el que le corresponde al 2, porque ambos difieren en el tercer dígito... y así sucesivamente. Es decir, no existe una correspondencia uno-a-uno porque el número real $x$ ha quedado fuera de la relación uno-a-uno. Por consiguiente, $\mathbb{R}$ no es numerable. Una consecuencia de este resultado es que los números reales no pueden contarse ya que no puede determinarse qué número real le sigue a un número real dado. Por ejemplo, ¿qué número real le sigue al 4? ¿Será 4,0001 0 4,000000000000000001 La respuesta es: ninguno de ellos. Además, nadie sabe, ni sabrá, cuál es el número que le sigue al debido a que cualquiera que sea la cantidad de ceros que se escriba después de la coma decimal y antes del 1 siempre se le podrá agregar otro cero.

Si relacionamos los números reales con el devenir del tiempo, entonces "al número dado" lo relacionamos con "este instante". Pero no sabemos, a ciencia cierta, cuál es "el instante siguiente", porque en cuanto pretendemos asirlo, el "instante siguiente" ya es otro. El conjunto de rock Los enanitos verdes grafica lo dicho en unos versos de su canción "La muralla" (1987), cuando dice “... estoy parado sobre la muralla que divide todo lo que fue de lo que será...”. Parafraseando, diríamos: Cada vez que queramos que algo sea... ya fue: Vivimos con un pie en el pasado y el otro en el futuro.

¡Además, se ha visto que el conjunto $\mathbb{Q}$ es numerable y como se cumple que $\mathbb{R}=\mathbb{Q}$ UI entonces el conjunto II necesariamente debe ser no numerable, porque, en caso contrario, el segundo miembro de la igualdad sería la unión de dos conjuntos numerables y, por tanto, $\mathbb{R}$ debería ser numerable, contradiciendo el resultado obtenido, que indica que no lo es. Esto significa que en $\mathbb{R}$ existen "más" números irracionales que números racionales, ya que estos últimos pueden contarse mientras que los primeros ni siquiera los podemos contar. La condición de conjunto no numerable de $\mathbb{R}$ depende de la no numerabilidad del conjunto II.

Por otro lado, se dice que un número real es algebraico si es la solución de una ecuación polinómica cuyos coeficientes son números racionales, y si no es algebraico entonces el número real se denomina trascendente ${ }^{10}$. Esto es, los números

10 El nombre trascendente se debe al matemático suizo Leonhard Euler (1707-1783), quien decía que estos números trascienden el poder de los métodos algebraicos, tal como cita Joaquín Navarro (2011) No tiene ninguna connotación filosófica adicional. 
trascendentes no pueden ser obtenidos directamente mediante la resolución de una ecuación polinómica con coeficientes racionales, sino como raíces de cualquier otro tipo de ecuación.

Por ejemplo, son números algebraicos todos los números racionales, incluyendo a los números naturales y a los números enteros, porque son raíces de ecuaciones que tienen la forma $a x+b=0$ También los números irracionales, tales como $\sqrt{3}$ y $\frac{1+\sqrt{5}}{2}$, son números algebraicos, porque el primero es raíz de la ecuación polinómica $x_{-}^{2}-3=0$, mientras que el segundo, llamado el número de oro, es raíz de la ecuación polinómica $\mathrm{x}^{2}-\mathrm{x}-1=0$.

Algunos números trascendentes destacados son $\pi, e, e^{\pi}, 2^{\sqrt{2}}$ (constante de Hilbert). Otros números trascendentes son el creado en 1945 por el matemático norteamericano Arthur Copeland (1898-1970) y el matemático húngaro Paul Erdös (1913-1996), llamado número de Copeland-Erdös: 0,2357111317... (El número consiste en todos los números primos a partir de 2); y el creado en 1933 por el matemático inglés David Chapernowne (1912-2000), llamado número de Chapernowne: $0,1234567891011 \ldots$ (El número está conformado por todos los números naturales).

Se demuestra que la adición de dos números algebraicos es otro número algebraico y que la suma de un número algebraico y un número trascendente es un número trascendente. Pero el resultado de la suma de dos números trascendentes puede ser 0 un número trascendente 0 un número algebraico. Por ejemplo, la suma de los números trascendentes $5+\pi \mathrm{y}-\pi$ y es igual al número algebraico $\sqrt{5}$. Además, la suma de los números trascendentes $\pi^{2}+\pi^{2}$ es un número trascendente. Sin embargo, no se sabe si , los dos números trascendentes más famosos, son racionales o irracionales, y, si son irracionales, si son trascendentes o algebraicos. La mayoría de los matemáticos supone que son irracionales y trascendentes, pero en matemáticas no basta ni el consenso ni las mayorías para decidir: se debe acudir a una demostración y no a una votación.

De acuerdo a los ejemplos presentados, se ve que los números racionales y algunos números irracionales son números algebraicos. El resto de números irracionales son números trascendentes. Entonces, parece obvio que hay más números algebraicos que números trascendentes. Pero esta afirmación es falsa, ya que Cantor demostró que el conjunto de números algebraicos es numerable (la demostración contiene detalles técnicos que embrollarían el texto), y como $\mathbb{R}=\mathbb{A} \cup \mathbb{T}$, se sigue que, aplicando un análisis similar al presentado para justificar que el conjunto II no es numerable, el conjunto $\mathbb{T}$ no es numerable. Es decir, hay más números reales trascendentes que números reales algebraicos. Los notables números (relaciona la longitud de una circunferencia con su diámetro) y (base de los logaritmos naturales) son números irracionales que pertenecen al conjunto no numerable de los números reales trascendentes, mientras que $\varphi$ (razón áurea, número de oro, número dorado,...) es un número irracional que pertenece al conjunto numerable de los números reales algebraicos.

El primero de los siguientes diagramas de Venn-Euler grafica la clasificación de los números reales respecto de la Numerabilidad/No numerabilidad y el segundo diagrama, respecto de lo Algebraico/Trascendente.
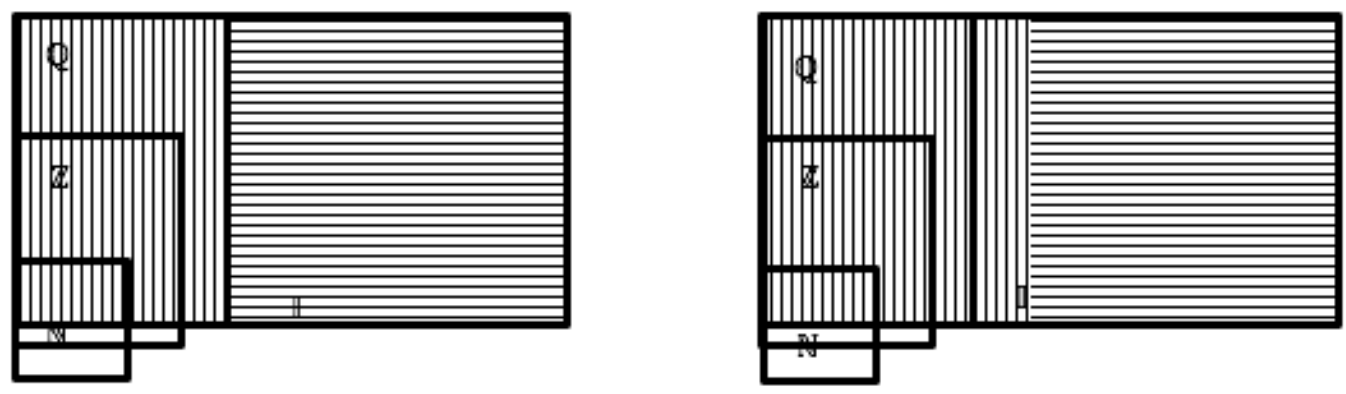

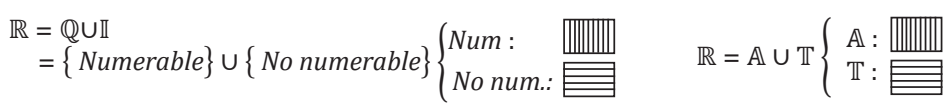

La saga de los números reales continúa. Esto, porque el matemático francés Émile Borel (1871-1956), en el año 1909, dio a conocer el concepto de número real normal. Este nuevo número es un número irracional, escrito en cualquier base, cuyos dígitos, considerando la parte entera y los que están después de la coma decimal, siguen una distribución uniforme en la 
presentación de los dígitos que la conforman, en el sentido de que las cifras $0 ; 1 ; 2 ; \ldots 9$, las combinaciones de dos en dos, de tres en tres,... de en dígitos, tienen igual probabilidad de aparición (por esto, debieron llamarse números uniformes, pero su creador prefirió números normales). Borel, recurriendo a la demostración por reducción al absurdo ${ }^{11}$, mostró que existen infinitos números normales. Sin embargo, no presentó un ejemplo de número normal. Sin embargo, el matemático polaco Waclaw Sierpinski (1882-1969) publicó el primer ejemplo de número normal en un artículo de la edición 45 del Bulletin de la Société Mathématique de France publicado en 1917. Otros números normales son el número de Copeland-Erdös y el número de Champernowne. No se sabe si los números y son normales.

Se demuestra que el conjunto de los números normales no es numerable y que abarcan a casi todo $\mathbb{R}$. Esto es, que la "mayoría" de los números reales son normales.

Aquí se deja este apasionante tema, a pesar de que hay más información sobre los números normales y otros tipos especiales de números reales, como los números computables o números construibles, entre otros, lo que significaría entrar en detalles técnicos que dificultarían la lectura de este documento. Para otra vez será.

- Respecto de la propiedad de clausura, los números reales la verifican para la adición, sustracción, multiplicación y división, pero no sucede lo mismo para la radicación de números reales, ya que, por ejemplo, $\sqrt{-4}$ no es un número real.

Los números complejos abarcan a los números reales y a los números imaginarios. El conjunto se denota por $\mathbb{c}$ siendo $\mathbb{C}=\{\alpha+b i / \alpha € \mathbb{R}, b € \mathbb{R}, \mathrm{i}=\sqrt{-1}\}$. La expresión $\alpha+b i$ es un número real si $b=0$, y es un número imaginario si $\alpha=0$. Como los números complejos también se pueden representar como pares ordenados de números reales (a;b), entonces su representación geométrica se realiza en un plano que recibe el nombre de plano de Argand, en recuerdo del matemático suizo Jean Argand (1768-1822), quien, en un ensayo publicado en 1806, postuló esta representación, que ya había sido sugerida en 1799 por el matemático noruego Caspar Wessel, aunque sin mucho éxito. En esencia, el número complejo es la respuesta a la pregunta ¿qué representa la raíz cuadrada de un número negativo?, cuya primera referencia documentada se remonta al siglo I, cuando el matemático griego Herón de Alejandría (aproximadamente entre 10 y 75 d.C.), en un cálculo realizado en su obra Stereometría, obtiene. Luego, por el año 275, el matemático griego Diofanto (aproximadamente entre 200 y 284), considerado el padre del álgebra, planteó resolver una ecuación con raíces complejas (un dato interesante acerca de este matemático es que, a pesar de no conocerse las fechas exactas de su nacimiento y muerte, por su epitafio ${ }^{12}$, escrito como un problema de álgebra, se sabe que vivió 84 años). Durante muchos años se trató de esquivar la respuesta a la pregunta, evitando que aparecieran en los cálculos, pero ante su presencia pertinaz no hubo otra posibilidad que enfrentarla. El matemático suizo Leonhard Euler (1707-1783) fue el primero en utilizar la notación $\mathrm{i}=\sqrt{-1}$, relacionando los números complejos con la función exponencial y las funciones trigonométricas del siguiente modo: $\mathrm{e}^{\mathrm{i} x}=\cos x+i s e n x$. Euler es el creador de la expresión matemática $e^{i \pi}+1=0$, considerada la más bella ${ }^{13}$ de las matemáticas, ya que contiene a las constantes matemáticas e, i, $\pi, 1,0$ en una relación muy sencilla.

Algunos números complejos son $1 ;-3 ; 5 / 7 ; \pi ; \sqrt{2} ; \varphi ; \sqrt{-4} ; ; 4+5 i ;-10 i$. En matemática se muestra que el conjunto $\mathbb{c}$ no es numerable, siguiendo un razonamiento parecido a la No numerabilidad de $\mathbb{R}$. Las operaciones de adición, sustracción, multiplicación, división y radicación de números complejos verifican la propiedad de clausura.

Obsérvese que se comenzó con el conjunto de los números naturales, en el cual no se verifica la propiedad de clausura para la sustracción y, a partir de ahí, se ha ido ampliando este conjunto hasta llegar al conjunto de los números complejos, en el cual todas las operaciones básicas verifican la propiedad de clausura.

En la vida cotidiana las personas utilizan los números para medir, contar y ordenar. Para estos procesos generalmente son necesarios los números naturales, enteros y racionales. Esta afirmación no niega que, a veces, en alguna actividad, se necesite de un número irracional, el cual será usado con un valor aproximado. Por ejemplo, $\pi$ (relación entre la longitud de una circunferencia y su diámetro) es un número irracional y, cada vez que es utilizado en algún cálculo, se utiliza el valor aproximado de 3,1416 0 3,14.

11 El matemático inglés G.H. Hardy (1877-1947) decía sobre la demostración por reducción al absurdo: "Se trata de una estrategia mucho más refinada que cualquier jugada de ajedrez: un ajedrecista se puede permitir sacrificar un peón o incluso alguna otra pieza, pero un matemático arriesga la partida entera".

12 "Transeúnte, esta es la tumba de Diofanto: los números pueden mostrar, joh maravilla!, la duración de su vida. Su niñez ocupó la sexta parte de su vida; después, durante la decimosegunda parte, de vello se cubrieron sus mejillas. Pasó aún una séptima parte de su vida antes de tomar esposa y, cinco años después, tuvo un precioso niño que, una vez alcanzada la mitad de la edad de su padre, pereció de una muerte desgraciada. Su padre tuvo que sobrevivirle, llorándole, durante cuatro años. De todo esto se deduce su edad" (Respuesta: la edad de Diofanto es 84 años. Algunas respuestas adicionales: se casó a los 26 años de edad, tuvo un hijo a los 38 años y el hijo vivió 42 años).

13 Clifflord Pickover, prolífico autor contemporáneo de libros sobre matemáticas, indica que la fórmula $\lim _{\eta \rightarrow \infty}\left(\frac{\mathrm{e}^{\eta} \eta !}{\eta^{\eta} \sqrt{\eta}}\right)=\sqrt{2 \pi}$, del matemático escocés James Stirling (1692-1770), tiene una belleza que conmueve a los matemáticos (en la fórmula aparecen diferentes operaciones del número natural $\mathrm{n}$ ). 
Los números complejos, en cambio, tienen pocas probabilidades de aparecer en los problemas del día a día que se refieran a medir, contar, ordenar y codificar objetos. Pero en la mecánica cuántica, electrónica y electricidad, entre otras áreas de las ciencias, su aplicación ha sido notoria. Incluso, se afirma que sin la presencia de estos números la energía eléctrica no existiría y aún estaríamos utilizando la energía no renovable del carbón, con todas las consecuencias que su uso conlleva.

\section{Números notables}

Algunos números tienen ciertas regularidades o características que los diferencian de los otros números. Estos números pueden pertenecer a cualquiera de los conjuntos numéricos presentados en el acápite anterior. Tal como dice Gabriel Navarro en Un curso de números (2007), "Algunas veces, cuando tratamos con números, no es sencillo distinguir lo que es matemáticamente relevante de lo que tiene un interés preferentemente lúdico". Estos dos aspectos, la relevancia y lo lúdico, han guiado la selección de los números notables que a continuación presentamos: primos, perfectos y amigos. Los tres números son conocidos desde la época de los griegos. Además, los números primos tienen un rol protagónico en la actualidad, ya que participan en la encriptación de la información para no hacer de conocimiento público lo que es confidencial 0 reservado; esto sucede, asimismo, en los números perfectos y amigos, por la relación que tienen con los números primos.

Los números irracionales, $\pi$, e y $\varphi$ y , tienen una enorme importancia en muchas de las aplicaciones de las matemáticas. Para presentarlas se necesitaría un libro 0, por lo menos, un artículo, el cual será presentado posteriormente.

Solamente mencionaremos a los números naturales que tienen un interés lúdico, que muy bien pueden llamarse números de fantasía. La siguiente lista de números no es exhaustiva: ambiciosos, apocalípticos, automorfos, casi perfectos, cíclicos, curiosos, hambrientos, infelices, intocables, felices, malvados, narcisistas, novios, oblongos, odiosos, omirp, piramidales, poderosos, raros, rectangulares, repunit, sociables, sordos, trimorfos, vampiros...

\section{Números primos}

Un número natural $\eta$, mayor que 1, se llama primo (o simple), cuando solamente tiene dos divisores: 1 y $\eta$. Y si tiene más de dos divisores, entonces el número se denomina compuesto. Por ejemplo, son números primos $2 ; \ldots 23 ; \ldots ; 313 . . . ;$ porque 2 es divisible solo por 1 , en tanto 2 y 23 son divisibles únicamente por 1 y 23; por otro lado, ...313 es divisible solamente por 1 y 313 mientras que son números compuestos: $4 ; 6 ; \ldots 144 ; \ldots$ ya que 4 tiene como divisores $1 ; 2$ y $4 ; 6$ tiene como divisores a $1 ; 2 ; 3$ y $6 ;. . .144$; tiene como divisores a $1 ; 2 ; 3 ; 4 ; 6 ; 8 ; 12 ; 18 ; 36 ; 72$ y 144.

Los números primos tienen importancia matemática, porque el Teorema Fundamental de la Aritmética, también llamado Teorema de Factorización Única, nos indica que cualquier número natural $\eta>$ tal como $\eta>1$ se puede escribir como un producto de potencias de números primos entre sí14. Haciendo un símil, puede afirmarse que los números primos conforman el ADN de los números. Por ejemplo, $120=2^{3} \times 3 \times 5,666=2 \times 3^{2} \times 37$.

El matemático griego Euclides (aproximadamente entre 330 a.C. y 275 a.C.), en su libro Elementos, demostró que no existe un número primo que sea mayor que todos los números primos. La demostración es una joya matemática que se inscribe en las del tipo de reducción al absurdo.

Al momento de escribir este artículo no se ha encontrado una función que genere todos los números primos y parece ser que su obtención seguirá retando al intelecto humano por muchos años más. Lo que se ha ido obteniendo son diversas funciones polinómicas, como las presentadas por Euler o por Adrien-Marie Legendre (1752-1833); o las funciones exponenciales, presentadas por el matemático francés Fermat (1601-1665) o por el sacerdote, filósofo y matemático francés Marin Mersenne (1588-1648). La función presentada por Mersenne es $M \eta=2 \eta-1$, siendo $\eta$ un número primo (esto último no es condición suficiente) ${ }^{15}$, merece una mención aparte porque es la que ha producido hasta ahora el mayor número primo y, como veremos después, el mayor número perfecto.

14 Los números naturales $m$ y $n$, que no son primos, se denominan primos entre sí siempre que el 1 es el único divisor común a $m$ y $n$. Ejemplos: 3 y 25,324 y $173, \ldots$

15 Si la condición "n es primo" fuera una condición suficiente, entonces, para probar que $2^{n}-1$ es un número primo, bastaría con mostrar que $\mathrm{n}$ es primo. Pero el número primo 11 produce al reemplazar en la expresión produce el número 2047 que no es primo porque 2047=23×89 (ver p. 19 de este articulo). 
Por otro lado, existen muchas conjeturas (afirmaciones que no se sabe si son verdaderas o falsas) referidas a resultados sobre los números primos. Entre estas se encuentran dos afirmaciones planteadas en 1742 por el matemático ruso Christian Goldbach (1790-1764), llamadas Conjetura fuerte (todo número par mayor de 2 puede escribirse como la suma de dos números primos) y Conjetura débil (todo número impar mayor que 5 puede escribirse como la suma de tres números primos). El matemático peruano Harald Helfgott, en 2013, ha mostrado que la segunda conjetura es verdadera, por lo que se ha convertido en un teorema. La resonancia de este logro matemático se ha limitado solamente al ámbito académico de la matemática: una conjetura menos y un teorema más. Lo hizo por el honor del espíritu humano ${ }^{16}$.

En la actualidad, los números primos son una herramienta poderosa para encriptar mensajes (esto es, cumplen la cuarta función de los números: codificar cosas). Dan una seguridad muy alta, porque descifrar un mensaje escrito utilizando algoritmos que contengan números primos grandes es muy laborioso, aunque, como lo menciona Joan Gómez en su libro, sobre codificación y criptografía, Matemáticos, espías y piratas informáticos (2010), no es imposible.

En el entretenido y divertido libro Matemática recreativa 1 se presenta el número primo capicúa 1000000000000066600000000000001 (por si acaso, hay veintiséis ceros repartidos en dos grupos de trece cada uno), denominado primo de Belfegor. Es un número capicúa porque si se lee de izquierda a derecha o de derecha a izquierda se obtiene el mismo número. 0jo, cuidado... Belfegor es un demonio conocido como el demonio de la pereza 0 el señor de la apertura, que seduce a las personas ofreciéndoles inventos ingeniosos que supuestamente proporcionan riquezas.

Entre los números primos, que ya son especiales, a partir del número primo 3 existen algunos números primos súper especiales: números primos consecutivos separados por un número par. Este tipo de números primos se denomina números primos gemelos. Los siguientes números primos son primos gemelos: y, 11 y 13, 17 y 19, 29 y 31 ... Pero también hay números primos gemelos "gigantescos", como los siguientes que tienen nada más que dígitos y fueron descubiertos en 2007.

Paolo Giordano (2008) ha escrito la novela La soledad de los números primos, que no tiene nada que ver con los números primos como tales, sino que es una historia romántica y triste acerca de Alice y Mattia, en la cual el autor hace un símil entre sus personajes y los números gemelos primos, porque dice que Mattia pensaba que él y ella eran como dos primos gemelos que estaban juntos pero no tanto como quisieran, porque siempre se interponía un "número par" entre ellos.

El mayor número primo que se ha determinado, por lo menos hasta julio de 2016, es el número el cual es un número primo de Mersenne. Fue descubierto por Curtis, Kurowski, Blosser y otros colaboradores pertenecientes a la red académica "Gran Búsqueda en Internet de los Primos de Mersenne" (GIMPS, por sus siglas en inglés, Great Internet Mersenne Prime Search). El descubrimiento se hizo público en el boletín de GIMPS, publicado en enero de 2016, en el cual se celebraban los veinte años de su creación.

\section{Números perfectos}

Un número natural $\eta$ es perfecto si la suma de sus divisores propios ${ }^{17}$ es igual a $\eta$. Estos números fueron estudiados por Pitágoras y sus seguidores pero más por un afán místico que por razones numéricas. Los números perfectos más pequeños son el 6 y el 28 porque la suma de sus divisores verifican la definición $(6=1+2+3$ y $28=1+2+4+7+14)$. Los siguientes dos números perfectos son el 496 y el 8128). Estos cuatro números ya eran conocidos por los griegos. Doscientos años después del análisis que efectuaran los pitagóricos sobre otras propiedades de estos números, como, por ejemplo, que los números perfectos siempre son iguales a la suma de una serie consecutiva de números cardinales ${ }^{18}$, tal como menciona Simon Sing en El enigma de Fermat (2015).

Euclides, en los Elementos, presentó el siguiente teorema, mediante el cual se generan números perfectos, "Si tantos números como se quiera, a partir de una unidad, se disponen en proporción duplicada hasta que su total resulte primo, y el total multiplicado por el último produce algún número, el producto será perfecto", que, escrita en el lenguaje matemático actual,

16 Ver parte final de Números perfectos, en la página 221 de este artículo.

17 Los divisores propios de un número natural $\eta$ son todos los números naturales mayores o iguales que 1 y menores que $\mathrm{n}$ tales que lo dividen exactamente, es decir el residuo es igual a cero. Ejemplo: Los divisores propios de 36 son: 1; 2; 3; 4; $6 ; 9 ; 12$ y 18; y los de 15 son: $1 ; 3$ y 5 . Además, todos los números primos tienen solamente a 1 como divisor propio.

18 Las relaciones son: $6=1+2+3 ; 28=1+2+3+4+5+6+7 ; 496=1+2+3+\ldots+30+31$; $8128=1+2+3+4+\ldots 126+127$. 
se traduce como: "Si la expresión $2^{\eta}-1$ es un número primo y $P=2^{\eta-1}\left(2^{\eta}-1\right)$, entonces $P$ es un número perfecto". Este teorema indica que cada vez que se obtiene un número primo de la forma $2^{n}-1$ (número primo de Mersenne), entonces, para generar un número perfecto, es suficiente con multiplicarlo por $2^{n-1}-1$. Más de 1500 años después, Euler demostró que todos los números perfectos pares $P$ pueden escribirse como $P=2^{\eta-1}\left(2^{\eta}-1\right)$, para un número natural $\eta$ adecuado. Por esto, al teorema mencionado, a veces se la conoce, como Teorema de Euclides-Euler.

Los cuatro números perfectos mencionados aparecen en el libro Introductio Aritmética de Nicómaco de Gerasa (aproximadamente entre 60-120). Parece que, basándose en esos resultados, efectuó varias conjeturas, algunas de las cuales se ha demostrado que son falsas, pero hasta el día de hoy han quedado dos: "Existe una cantidad infinita de números perfectos", y "No existen números perfectos impares". Además, en el libro presentó la clasificación de los números en: a) números abundantes (la suma de sus divisores propios es mayor que el número; por ejemplo, el 12, ya que la suma es 16); b) números deficientes (la suma de sus divisores propios es menor que el número; por ejemplo, el 16, puesto que la suma de sus divisores propios es 15 , y es que todos los números primos son deficientes, pues la suma de los divisores propios es) y c) números perfectos (la suma de sus divisores propios es igual que el número; por ejemplo, el 6 es perfecto, porque la suma es, precisamente 6).

Nicómaco le da a su clasificación numérica una connotación moral, afirmando que, en el caso de los números abundantes, se está hablando de excesos, exageraciones e, incluso, abusos; en el caso de los números deficientes, uno se refiere a privaciones, insuficiencias, deseos no satisfechos, pero, para el caso de los números perfectos, la referencia es la mesura, decoro, belleza y, por último, virtud.

Transcurrieron cientos de años hasta que el matemático alemán Johan Müller (1436-1476), más conocido como 'Regiomontano', descubriera los dos siguientes números perfectos. Esto se evidencia en los manuscritos escritos por Müller, alrededor de 1460. Estos son $2^{12}\left(2^{13}-1\right)=33550336$ y $2^{16}\left(2^{17}-1\right)=8589869056$. El quinto número rechaza la afirmación de Gerasa, quien decía que este número perfecto debía tener cinco dígitos, y el sexto, la afirmación de que los números perfectos, alternadamente, terminan en 6 o en 8 y, de acuerdo a esta afirmación, el sexto número perfecto debía terminar en 8.

Durante mucho tiempo se creyó que si $\eta$ era primo, entonces $2^{\eta}-1$ tenía que ser primo, pero, en 1536, el matemático Uldarico Regius mostró que para el número primo 11 esto no es cierto, porque $2^{11}-1=2047=23 \times 89$. Esto es, $2^{11}-1$ no es primo.

El tamaño de los números perfectos ha ido creciendo, ya que el mejoramiento de los algoritmos usados y de las tecnologías aplicadas han hecho posible de que Curtis, Kurowski, Blosser y otros hayan obtenido el $49^{\circ}$ número primo de Mersenne ${ }^{19}$ : $2^{74207281}-1$, y, por ende, el número perfecto $2^{74207280}\left(2^{74207281}-1\right)$ que hasta julio de 2016 es el número perfecto más grande.

Simon Sing (2015) afirma que San Agustín de Hipona (354-430), en La Ciudad de Dios, "sostiene que, aunque Dios podía haber creado su obra en un instante, decidió hacerlo en seis días para poner de manifiesto la perfección del mundo...El es un número perfecto en sí mismo y no porque Dios creara todas las cosas en seis días para que ese número sea perfecto. Y continuaría siéndolo incluso si la obra de los seis días no existiera." Además, el siguiente número perfecto es el 28, que coincide con el ciclo menstrual/lunar. Por otro lado, el religioso, filósofo y matemático inglés Alcuin de York (730-804), quien introdujo el Trivium y el Quadrivium, manifiesta que el segundo origen de la raza humana (a partir del Diluvio Universal) partió del número deficiente 8, pues ocho fueron los sobrevivientes humanos: Noé, sus tres hijos y sus respectivas esposas. Así que la culpa de todo lo malo que le sucede a la humanidad, a partir de ese episodio bíblico, lo tiene el número deficiente 8. También se afirmaba, en el siglo XII, que el estudio de los números perfectos ayudaba a "sanar las almas" (?).

El divulgador científico y filósofo de las ciencias Martin Gardner (1914-2020) decía que “...es complicado encontrar un conjunto de números naturales con una historia más fascinante y con propiedades rodeadas de profundos misterios, pero a su vez más inútiles que los números perfectos". El matemático y físico inglés Peter Barlow (1776-1862), en su libro Theory of Numbers (1811), afirmó que los números perfectos son simplemente curiosidades sin ninguna utilidad. Y posiblemente no le falte razón. Pero, al respecto, hay que recordar al matemático alemán Carl Jacobi (1804-1851), quien, en una carta enviada al matemático francés Adrien-Marie Legendre (1752-1833), le decía, entre otras cosas, lo siguiente: "M. Fourier ${ }^{20}$ opinaba que la finalidad primordial de las matemáticas consistía en su utilidad pública y en la explicación de los fenómenos naturales; pero un filósofo como él debería haber sabido que la finalidad única de la ciencia es la de rendir honor al espíritu humano y que por ello una cuestión sobre números vale tanto como una cuestión sobre el sistema del mundo" (La negrita no es de Jacobi).

19 Ver final de Números primos, de la página 220 de este artículo.

20 Matemático francés Jean-Baptiste Fourier (1768-1830). 


\section{Números amigos}

Dos números naturales $p$ y $q$ son amigos si la suma de los divisores propios de $p$ es igual a $q$ y, viceversa, la suma de los divisores propios de q es igual a p. El filósofo neopitagórico Jamblico (aproximadamente entre los años 245 y 330 ) atribuye el descubrimiento de los números amigos al mismo Pitágoras, indicando que, al preguntársele al filósofo "¿qué es un amigo?", este respondió: "Álter ego"21. La analogía matemática se refiere a dos números que comparten mutuamente la adición de sus divisores propios.

El par de números amigos de menor tamaño son 220 y 284 ya que se cumple: $220=1+2+4+71+142$ y $284=1+2+4+$ $5+10+11+20+22+44+55+110$, siendo los sumandos de 220 los divisores propios de 284 , y los sumandos de los divisores propios de 220. Estos números ya eran conocidos por Pitágoras y los miembros de su escuela. Obsérvese que, curiosamente, el número 220 es abundante y el número 284 es deficiente.

El matemático y astrónomo turco Thabit ibn Qurrá ibn Marwan al-Sabi al-Harrani (836-901 d.C.), quien, según Copérnico, determinó que la rotación de la Tierra alrededor del Sol duraba 365 días, 6 horas, 9 minutos y 12 segundos (tan solo un error de 2 segundos en un cálculo realizado manualmente, sin apoyo de la tecnología), obtuvo, aproximadamente en el año 850 d.C., una regla para generar números amigos, con la cual se obtenían los tres primeros pares de números amigos. En Europa, la Regla de Tabit no fue conocida, de modo que cuando, en 1636, Fermat "descubrió" el segundo par (17 296; 18 416) y, en 1638, Descartes "descubrió" el tercer par (9 363 584; 9437 056) fue porque ambos redescubrieron la Regla de Tabit. Estos descubrimientos fueron documentados en cartas enviadas por los mencionados matemáticos al sacerdote Marin Mersenne. Cuando interviene Euler en este tema, en 1737, solamente se conocían tres pares de números amigos, los cuales habían sido descubiertos en dos mil años. Luego, en 1747, Euler presentó nada menos que una lista de 62 pares de números amigos. No desmerece su grandioso logro matemático el que se hayan descubierto que dos parejas de su lista no son números amigos. En su investigación concibió la llamada Regla de Euler, para generar números amigos que tienen como caso particular a la Regla de Tabit. Un dato anecdótico sucedió en 1866, cuando el joven italiano Niccolo Paganini (no confundir con el eximio violinista), estudiante de secundaria de 16 años de edad, descubrió el segundo par de números amigos más pequeño: 1184; 1210. Esta pareja de números amigos, tan cercana a la primera pareja, se les "escapó" a los griegos, árabes y a todos los matemáticos de renombre, como Fermat, Descartes, Euler y otros, quienes, sistemáticamente, buscaban añadir otras parejas a las ya obtenidas.

La búsqueda de pares de números amigos continúa. Lo que ha cambiado es la forma de hacerlo, porque hasta pasada la mitad del siglo pasado se realizaba de manera manual. Pero, en nuestra época, regida por las computadoras, la búsqueda se realiza aplicando la sofisticación tecnológica con que cada mañana nos sorprende Silicon Valley. De modo que puede afirmarse que cuando se hacía la búsqueda en forma manual, su hallazgo era algo parecido a encontrar pepitas de oro, pepitas que ahora se están convirtiendo en granos de arena, ya que, a la fecha, hay como doce millones de parejas de números amigos.

En la página www.vaxasoftware.com² se presenta una lista de 142 pares de números amigos, desde la conformada por los números 220 y 284 hasta la conformada por 18655744 y 19154336.

Al igual que para los números primos y perfectos, los números amigos tienen tres conjeturas que están pendientes de ser demostradas: i) Las parejas de números amigos son infinitas, ii) Los números que conforman una pareja de números amigos siempre tienen la misma paridad, y iii) Los números que conforman una pareja de números amigos impares siempre son divisibles por tres.

Como dato curioso, mencionaremos que en la Edad Media se vendían talismanes tallados con los números amigos 220 y 284, porque creían que, de este modo, se protegía el amor entre las personas que los llevaban. Esto era una extensión de lo que los árabes practicaban, ya que grababan el 220 en una fruta y el 284 en otra fruta de la misma especie. Luego comían una de ellas y ofrecían la segunda a la elegida de su corazón. En estos casos, los números perfectos tenían la función de ser las flechas de Cupido.

Finalmente, si un número es perfecto entonces es un número amigo de sí mismo. Esto nos recuerda el aforismo del novelista inglés R.L. Stevenson (1850-1894): "Un amigo es una imagen que tienes de ti mismo".

21 Álter ego: locución latina que puede traducirse como 'el otro yo'; una persona en que se tiene confianza absoluta y con la que comparte todo.

22 www.vaxasoftware.com/doc_edu/mat/numamigos_esp.pdf 


\section{Bibliografía}

Courant, R., y RobBins, H. (1979 [1941]). ¿Qué son las matemáticas? Una exposición elemental de sus ideas y métodos (trad. L. Bravo Gala). Madrid: Aguilar. Recuperado de http://www.cimat.mx/ gil/docencia/2010/elementales/ que_es_la_matematica.pdf

García del CID, L. (2011). Números notables. Barcelona: RBA Libros.

Gracián, E. (2011). Un descubrimiento sin fin. El infinito matemático. Barcelona: RBA Libros.

Lıvı, M. (2009). ¿Es Dios un matemático? (trad. F. Pedrosa Martín). Barcelona: Ariel.

Navarro, J. (2011). Los secretos del número $\pi$. ¿Por qué es imposible la cuadratura del círculo? Barcelona: RBA Libros.

PiñelRo, G. E. (2013). Cantor. El infinito en matemáticas. Lo incontable es lo que cuenta. Navarra: RBA.

Singh, S. (2015). El enigma de Fermat (trad. D. Galadí y J. Gutiérrez). Barcelona: Ariel-Planeta.

Stewart, I. (2009). Historia de las matemáticas. En los últimos 10000 años. Barcelona: Crítica.

Tammet, D. (2015). La poesía de los números. Cómo las matemáticas iluminan mi vida (trad. P. Álvarez Ellacuria). Barcelona: Blackie Books.

Tang Fernández B., Contreras, P., Gálvez, H., Gálvez, R., y Núñez, L. (2012). Matemática recreativa. Lima: Fondo Editorial de la Universidad de Ciencia y Humanidades (UCH).

ZaLdivar, F. (2012). Introducción a la teoría de números. México, D. F.: Fondo de Cultura Económica. 